\title{
論説
}

\section{Nominal Idiosyncratic Shocks and Optimal Monetary Policy}

\section{Eisei Ohtaki}

\section{Introduction}

A characterization of optimal monetary policy is one of central issues in monetary theory. The traditional monetary theory has emphasized optimality of nonexpansionary monetary policy such as the Friedman rule (Friedman, 1969), which is one of the most famous dicta on monetary policy. ${ }^{1}$ In practice, however, there seems no central bank stating that its object is to implement such a nonexpansionary policy. Additionally, after the Lehman crisis in 2008, central banks of not a few developed countries adopted an expansionary monetary policy such as quantitative easing.

Differently from the traditional theory, recent studies on optimal monetary policy has demonstrated, in several environment, suboptimality of nonexpansionary monetary policy. As argued by Akyol (2004) and Kryvtsov, Shukayev, and Ueberfeldt (2011) for example, we now know that one of important sources of suboptimality of nonexpansionary policy is endowment (and productivity) shocks, which is uninsurable due to incompleteness of markets. Although these studies directly assumes the presence of endowment shocks, such shocks can be described endogenously. In the overlapping generations (OLG) model with spatial separation (Smith, 2002; Gomis-Porquears and Smith, 2003; Bhattacharya, Haslag, and Martin, 2005; Haslag and Martin, 2007; Bhattacharya, Haslag, and Martin, 2009) and search (Zhu, 2008), for example, endowment shocks arise endogenously and nonexpansionary policy becomes suboptimal. Then, the aim of

\footnotetext{
${ }^{1}$ The Friedman rule proposes the reducing policy to make the net rate of nominal interest rate zero.
} 
this article is to present another situation that nonexpansionary policy becomes suboptimal.

This article develops a simple OLG model of money with nominal idiosyncratic shocks. At each date, a new generation consisting of a continuum of ex-ante identical agents living for two periods is born. They also learn their types at the beginning of the second period of their lives. The nominal idiosyncratic shocks are then modeled by random money transfer, contingent upon agents' types in the second period, proportional to money holding. Although these shocks may be caused endogenously by, for example, money search and random relocation, we describe them as if they are exogenous nominal taxes/subsidies. In this model, Pareto optimality, which includes golden rule optimality, is adopted as an optimality criterion for stationary feasible allocations, or a first-best situation.

In this article, we characterize a monetary equilibrium by a difference equation and find an equivalent condition for the existence of a unique stationary monetary equilibrium given each money growth rate. It is shown that, for any money growth rate, the stationary monetary equilibrium given it does not realize a first-best situation, i.e.: it cannot generate a Pareto optimal allocation. So, we should consider a second-best situation. A second-best money growth rate is defined as one maximizing the welfare of stationary monetary equilibrium. We then show that a second-best rate of money growth exists and is greater than one. This implies suboptimality of nonexpansionary policy. A numerical example is also provided.

This article contributes to the literature by demonstrating suboptimality of nonexpansionary monetary policy under nominal idiosyncratic risks. Remark that, differently from the previous studies, our model does not impose any frictions such as spatial separation and search (except for friction due to the OLG framework). The important role of the nominal idiosyncratic shocks in our model is to cause the unexpected and nondegenerate ex-post distribution of money. In our model, nominal idiosyncratic shocks are (implicitly) assumed to be not insurable by the lack of complete asset structures and any other insurance instruments such as insurance companies. Therefore, such uninsured shocks causes heterogeneous money holdings among second-period agents in the same cohort and, as a result, endowment shocks.

This article then concludes that, even in the absence of any frictions, there exists a situation wherein expansionary policy can be optimal (in the sense of second best) and therefore frictions emphasized in the previous studies might not be critical components to make expansionary policy optimal. By the way, in our model, an ex-post nondegenerate distribution of money, 
caused by the exogenous nominal idiosyncratic risks, has an important role for optimality of expansionary policy. In the previous studies, such an ex-post nondegenerate distribution of money (between buyers and sellers in Zhu (2008) and between movers and nonmovers in Smith (2002) and others) is generated endogenously. One may consider that this is a main reason for suboptimality of reducing policy in the previous studies.

The organization of this paper is as follows: Section 2 introduces the primitives of the model that we consider. Section 3 presents our main results. Proofs of propositions are provided in Section 4 .

\section{The Model}

We develop a stationary overlapping generations model of money, wherein agents face idiosyncratic nominal shocks. Time is indexed by $t$ and runs discretely from 1 to infinity. In each period, there exists a single perishable commodity called the consumption good.

In each period, one new generation, consisting of a continuum of agents with unit mass, is born and lives for two periods. Agents are ex-ante identical but learn their type $\theta \in \Theta$ at the beginning of the second period of their lives, where $\Theta:=\{-\tau, \rho\}, 0<\tau \leq 1$, and $0<\rho$. The ex-post distribution of old agents on $\Theta$ is represented by a probability measure $\pi$ on $\Theta .^{2}$ Assume that $\sum_{\theta \in \Theta} \theta \pi_{\theta}=0$, which implies that $\pi_{\theta}>0$ for each $\theta \in \Theta$ and $\tau=\rho \pi_{\rho} /\left(1-\pi_{\rho}\right){ }^{3}$

The endowment and preference structures are independent of time and agents' types. Each agent is endowed with $\omega^{y}>0$ units of the consumption good in the first period of her life and $\omega^{o} \geq 0$ units in the second period. He/she ranks his/her consumption streams contingent upon their types in the second period, $c=\left(c^{y},\left(c^{\theta}\right)_{\theta \in \Theta}\right) \in \Re_{+} \times \Re_{+}^{\Theta}$, according to a lifetime utility function $U: \Re_{+} \times \Re_{+}^{\Theta} \rightarrow \Re$, where $c^{y}$ is first-period consumption and $c^{\theta}$ is second-period consumption when his/her type is $\theta$. It is assumed that there exist real-valued functions $u_{y}$ and $u_{o}$ on $\Re_{+}$such that, for each $\left(c^{y},\left(c^{\theta}\right)_{\theta \in \Theta}\right) \in \Re_{+} \times \Re_{+}^{\Theta}$,

$$
U\left(c^{y},\left(c^{\theta}\right)_{\theta \in \Theta}\right)=u_{y}\left(c^{y}\right)+\sum_{\theta \in \Theta} u_{o}\left(c^{\theta}\right) \pi_{\theta},
$$

where $u_{i}, i \in\{y, o\}$, is assumed to be strictly monotone increasing, strictly concave, and twice continuously differentiable on the interior of its domain. Also assume that $\lim _{x \downarrow 0} u_{i}^{\prime}(x)=\infty$ for each $i \in\{y, o\}$.

\footnotetext{
${ }^{2}$ The realizations of types are assumed to be identical and independent for agents in each generation.

${ }^{3}$ The model degenerates into the one without idiosyncratic nominal risk if and only if $\tau=\rho=0$. In order to consider the case with idiosyncratic risks, therefore, we have assumed that $\tau>0$. On the other hand, the proviso that $\tau<1$ is a further technical assumption to ensure the consistency in the model.
} 
In the initial period, there also exists a continuum of one-period-lived agents, called the initial olds, with unit mass. They have learned their own types $\theta \in \Theta$ and their distribution on $\Theta$ is represented by $\pi$, defined as above. Each of them is endowed with $\omega^{o}$ units of the consumption good and ranks his/her consumption $c_{1}^{\theta}$ according to $\bar{u}\left(c_{1}^{\theta}\right):=c_{1}^{\theta}$ when his/her type is $\theta \in \Theta$.

We adopt Pareto optimality as a criterion of optimality. A stationary feasible allocation of this economy is a vector $\left.c=\left(c^{y},\left(c^{\theta}\right)_{\theta \in \Theta},\left(c_{1}^{\theta}\right)_{\theta \in \Theta}\right)\right) \in \Re_{+} \times \Re_{+}^{\Theta} \times \Re_{+}^{\Theta}$ satisfying that $c^{y}+$ $\sum_{\theta \in \Theta} c_{1}^{\theta} \pi_{\theta}=\omega^{y}+\omega^{o}$ and $c^{y}+\sum_{\theta \in \Theta} c^{\theta} \pi_{\theta}=\omega^{y}+\omega^{o}$, where $c^{y}, c^{\theta}$, and $c_{1}^{\theta}$ are consumptions by the young agents, the type $\theta$ old agents, and the type $\theta$ initial olds, respectively. It is

- interior if it satisfies that $c^{y}>0$ and $c^{\theta}>0$ for each $\theta \in \Theta$,

- Pareto optimal (PO) if there is no stationary feasible allocation $\tilde{c}$ such that $U\left(\tilde{c}^{y},\left(\tilde{c}^{\theta}\right)_{\theta \in \Theta}\right) \geq$ $U\left(c^{y},\left(c^{\theta}\right)_{\theta \in \Theta}\right)$ and $\tilde{c}_{1}^{\theta} \geq c_{1}^{\theta}$ for each $\theta \in \Theta$ with strict inequality somewhere, and

- golden rule optimal (GRO) if it satisfies that $U\left(c^{y},\left(c^{\theta}\right)_{\theta \in \Theta}\right) \geq U\left(\tilde{c}^{y},\left(\tilde{c}^{\theta}\right)_{\theta \in \Theta}\right)$ for each stationary feasible allocation $\tilde{c}$.

Note that, for each stationary feasible allocation $c$, it must hold that $\sum_{\theta \in \Theta} c^{\theta} \pi_{\theta}=\omega^{y}+\omega^{o}-c^{y}=$ $\sum_{\theta \in \Theta} c_{1}^{\theta} \pi_{\theta}$ but it is not necessarily required that $c^{\theta}=c_{1}^{\theta}$ for each $\theta \in \Theta$.

In this economy, the central bank issues money, which is an infinitely-lived outside asset yielding no dividend. Its stock at date $t$ is denoted by $M_{t}$. The initial money stock $M_{0}$ (in the implicitly defined period 0) is given and held by the initial olds, where it is assumed that each initial old has $M_{0}$ units of money. Then, the constant money growth is assumed, i.e.: $M_{t}=\sigma M_{t-1}$ for each date $t \geq 1$, where $\sigma>0$. The newly issued money of period $t \geq 1$, $Z_{t}:=M_{t}-M_{t-1}$, is equally distributed among the old agents at the period. We also introduce idiosyncratic nominal shocks. An agent holding one unit of money at the beginning of each period will pay [resp. receive] $|\theta|$ units of money in that period if his/her type is $\theta<0$ [resp. $\theta \geq 0$ ]. Therefore, a type $\theta$ agent might be interpreted as a taxpayer if $\theta<0$ and a recipient if $\theta \geq 0$.

We denote by $p_{t}$ and $q_{t}$ the real price of money and the real money balance in period $t \geq 1$, respectively. Of course, it must hold that $q_{t}=p_{t} M_{t}$ for each period $t \geq 1$. Therefore, we define an equilibrium in terms of real money balances instead of real prices of money. A monetary equilibrium given money growth rate $\sigma$ is a positive sequence of real money balances, $q^{e}$, such that there exists a sequence $m^{e}$ such that: an initial old whose type is $\theta \in \Theta$ consumes 
$c_{1}^{\theta}=\omega^{o}+\left(q_{1}^{e} / M_{1}\right)\left((1+\theta) M_{0}+Z_{1}\right)$ and, for any period $t \geq 1$, (i) each young agent chooses his/her money holding $m_{t}^{e}$ to maximize $U\left(c_{t}^{y},\left(c_{t+1}^{\theta}\right)_{\theta \in \Theta}\right)$ subject to the first-period budget constraint,

$$
c_{t}^{y}=\omega^{y}-\left(q_{t}^{e} / M_{t}\right) m_{t}^{e},
$$

and the second-period budget constraint when his/her type is $\theta \in \Theta$,

$$
c_{t+1}^{\theta}=\omega^{o}+\left(q_{t+1}^{e} / M_{t+1}\right)\left((1+\theta) m_{t}^{e}+Z_{t+1}\right)
$$

and (ii) $m_{t}^{e}=M_{t}$. Moreover, it is stationary if there exists some $q>0$ such that $q_{t}^{e}=q$ for each $t \geq 1$. This is a standard definition of monetary equilibrium: condition (i) is the utilitymaximizing problem with sequential budget constraints and condition (ii) is the money market clearing condition. Note that both tax and subsidy are dominated by money and yield ex-post heterogeneity of money holding.

Although money grows and agents faces nominal idiosyncratic risks in our model, they do not affect feasibility of monetary equilibrium allocations. To be more precise, we can observe the following statement.

Remark. The good market equilibrium holds at any monetary equilibrium given any $\sigma>0$.

To verify this remark, let $\sigma>0$ and let $q^{e}$ be a stationary monetary equilibrium given $\sigma$. The consumption $\left\{\left(c_{t}^{y},\left(c_{t}^{\theta}\right)_{\theta \in \Theta}\right)\right\}_{t \geq 1}$ corresponding to $q^{e}$ is then given by $c_{t}^{y}=\omega^{y}-q_{t}^{e}$ and $c_{t}^{\theta}=\omega^{o}+\left(1+\theta \sigma^{-1}\right) q_{t}^{e}$ for each $t \geq 1$ because $m_{t}^{e}=M_{t}$ for each $t \geq 1$. Therefore, for each $t \geq 1$,

$$
\begin{aligned}
c_{t}^{y}+\sum_{\theta \in \Theta} c_{t}^{\theta} \pi_{\theta} & =\left(\omega^{y}-q_{t}^{e}\right)+\sum_{\theta \in \Theta}\left(\omega^{o}+\left(1+\theta \sigma^{-1}\right) q_{t}^{e}\right) \pi_{\theta} \\
& =\omega^{y}+\omega^{o}+\sigma^{-1} q_{t+1}^{e} \sum_{\theta \in \Theta} \theta \pi_{\theta} \\
& =\omega^{y}+\omega^{o}
\end{aligned}
$$

because $\sum_{\theta \in \Theta} \theta \pi_{\theta}=0$. This establishes the previous remark. As a summary, the money growth and nominal idiosyncratic risks themselves do not lose any amount of the consumption good.

\section{Main Results}

This section provides our main results. Proofs of those results are provided in the following section. Although we will consider the welfare of a stationary monetary equilibrium, we have adopted Pareto optimality (PO) as an optimality criterion for stationary feasible allocations. 
To apply the PO criterion to allocations corresponding to a stationary monetary equilibrium, we first explore a tractable condition identifiable with PO (and golden rule optimality, GRO).

Proposition 1 An interior stationary feasible allocation $c=\left(c^{y},\left(c^{\theta}\right)_{\theta \in \Theta},\left(c_{1}^{\theta}\right)_{\theta \in \Theta}\right)$ is Pareto optimal if and only if there exists some $c^{\circ}>0$ such that:

(A) $c^{o}=c^{\theta}$ for each $\theta \in \Theta$; and

(B) $u_{o}^{\prime}\left(c^{o}\right) / u_{y}^{\prime}\left(c^{y}\right) \leq 1$.

Furthermore, it is golden rule optimal if and only if there exists some $c^{o}>0$ satisfying $(A)$ and (B) with equality.

By this proposition, we can say that, at each PO allocation, (A) consumptions must fully insured and (B) the marginal rate of substitution between the first- and the second-period consumption must be less than or equal to one. Furthermore, GRO implies PO but PO does not necessarily imply GRO. This is standard characterizations of PO and GRO in the OLG model. ${ }^{4}$

We next explore equivalent condition for existence and uniqueness of stationary monetary equilibrium given a money growth rate. Before doing so, we characterize a monetary equilibrium by a difference equation.

Proposition 2 A positive sequence $q^{e}$ such that $q_{t}^{e} \in\left(0, \omega^{y}\right)$ for each $t \geq 1$ is a monetary equilibrium given money growth rate $\sigma>0$ if and only if it holds that

$$
\sigma q_{t}^{e} u_{y}^{\prime}\left(\omega^{y}-q_{t}^{e}\right)=q_{t+1}^{e} \sum_{\theta \in \Theta}(1+\theta) u_{o}^{\prime}\left(\omega^{o}+\left(1+\theta \sigma^{-1}\right) q_{t+1}^{e}\right) \pi_{\theta}
$$

for each $t \geq 1$.

As a corollary of this proposition, we can characterize a stationary monetary equilibrium, denoted by $q \in] 0, \omega^{y}[$, given $\sigma>0$ by the single equation:

$$
\sigma u_{y}^{\prime}\left(\omega^{y}-q\right)=\sum_{\theta \in \Theta}(1+\theta) u_{o}^{\prime}\left(\omega^{o}+\left(1+\theta \sigma^{-1}\right) q\right) \pi_{\theta} .
$$

To avoid the situation that the return of money at a stationary monetary equilibrium becomes negative, we will concentrate our attention on the situation that $\sigma \geq \tau$. We should then explore the condition for existence of a unique solution of this equation. The following proposition provides such a condition.

\footnotetext{
${ }^{4}$ Interested readers might find Ohtaki (2013), which characterizes golden rule optimality in a stochastic OLG environment.
} 
Proposition 3 For each money growth rate $\sigma \geq \tau$,

a. a stationary monetary equilibrium given $\sigma$ exists if and only if $r_{0}<1 / \sigma$; and

b. it is unique,

where $r_{0}:=u_{y}^{\prime}\left(\omega^{y}\right) / u_{o}^{\prime}\left(\omega^{o}\right)$.

By Proposition 1, the equivalent condition provided in Proposition 3.a can be interpreted as Pareto suboptimality of the initial endowment stream when $\sigma=1$. Given the initial endowment stream $\left(\omega^{y}, \omega^{o}\right)$, therefore, a stationary equilibrium is hard to exist when a money growth rate increases (and it banishes when $\sigma=r_{0}^{-1}$ ). We denote by $q_{*}(\sigma)$ the stationary monetary equilibrium given $\sigma \in\left[\tau, r_{0}^{-1}\right.$. Because a stationary monetary equilibrium exists and is unique for each $\sigma \in\left[\tau, r_{0}^{-1}\left[, q_{*}(\sigma)\right.\right.$ is well-defined. By applying the implicit function theorem, one can obtain that $q_{*}^{\prime}(\sigma)<0$ on its domain. Furthermore, it is easy to verify that $\lim _{\sigma \uparrow r_{0}^{-1}} q_{*}(\sigma)=0$.

We are now ready to argue on the optimal monetary policy. We first verify that the stationary monetary equilibrium given a money growth rate is never first-best, i.e.: it does not generate Pareto optimal allocation.

Proposition 4 For any $\sigma \in\left[\tau, r_{0}^{-1}[\right.$, the stationary monetary equilibrium given $\sigma$ cannot generate Pareto optimal allocations.

As shown in this proposition, the stationary monetary equilibrium given each money growth rate does not achieve the first-best situation. Therefore, we should consider the second-best situation. Given a money growth rate $\sigma$, the equilibrium welfare, denoted by $W(\sigma)$, can be written as

$$
W(\sigma)=u_{y}\left(\omega^{y}-q_{*}(\sigma)\right)+\sum_{\theta \in \Theta} u_{o}\left(\omega^{o}+\left(1+\theta \sigma^{-1}\right) q_{*}(\sigma)\right) \pi_{\theta} .
$$

In this article, we define a second-best situation as a situation wherein a money growth rate is chosen to maximize the welfare at stationary monetary equilibrium. To be more precise, a money growth rate $\sigma \in\left[\tau, r_{0}^{-1}\left[\right.\right.$ is second-best if $W(\sigma) \geq W(\tilde{\sigma})$ for each $\tilde{\sigma} \in\left[\tau, r_{0}^{-1}[\right.$.

To study a second-best money growth rate, define the function $V: \Re_{++}^{2} \rightarrow \Re$ by

$$
V(\sigma, q):=u_{y}\left(\omega^{y}-q\right)+\sum_{\theta \in \Theta} u_{o}\left(\omega^{o}+\left(1+\theta \sigma^{-1}\right) q\right) \pi_{\theta} .
$$

The welfare at the stationary monetary equilibrium given $\sigma, W(\sigma)$, can be then represented by $W(\sigma)=V\left(\sigma, q_{*}(\sigma)\right)$. By differentiating $W$, we can obtain that $W^{\prime}(\sigma)=V_{1}\left(\sigma, q_{*}(\sigma)\right)+$ 
$V_{2}\left(\sigma, q_{*}(\sigma)\right) q_{*}^{\prime}(\sigma)$, i.e.: an increment of the equilibrium welfare by an increase in $\sigma$ can be divided into two parts: $V_{1}\left(\sigma, q_{*}(\sigma)\right)$ and $V_{2}\left(\sigma, q_{*}(\sigma)\right) q_{*}^{\prime}(\sigma)$. By easy calculation, we can obtain that

$$
\begin{aligned}
V_{1}(\sigma, q) & =-\frac{q}{\sigma^{2}} \sum_{\theta \in \Theta} \theta u_{o}^{\prime}\left(\omega^{o}+\left(1+\theta \sigma^{-1}\right) q\right) \pi_{\theta} \\
& =-\frac{q}{\sigma^{2}}\left[\sum_{\theta \in \Theta^{-}} \theta u_{o}^{\prime}\left(\omega^{o}+\left(1+\theta \sigma^{-1}\right) q\right) \pi_{\theta}+\sum_{\theta \in \Theta^{+}} \theta u_{o}^{\prime}\left(\omega^{o}+\left(1+\theta \sigma^{-1}\right) q\right) \pi_{\theta}\right] \\
& >-\frac{q}{\sigma^{2}} u^{\prime}\left(\omega^{o}+q\right) \sum_{\theta \in \Theta} \theta \pi_{\theta}=0
\end{aligned}
$$

for each $\sigma \geq \underline{\theta}$ and each $q>0$, where $\Theta^{-}:=\{\theta \in \Theta: \theta<0\}$ and $\Theta^{+}:=\{\theta \in \Theta: \theta \geq 0\}$. We can also obtain that

$$
\begin{aligned}
V_{2}\left(\sigma, q_{*}(\sigma)\right) & =-u_{y}^{\prime}\left(\omega^{y}-q_{*}(\sigma)\right)+\sum_{\theta \in \Theta}\left(1+\theta \sigma^{-1}\right) u_{o}^{\prime}\left(\omega^{o}+\left(1+\theta \sigma^{-1}\right) q_{*}(\sigma)\right) \pi_{\theta} \\
& =\sum_{\theta \in \Theta}\left[-\frac{1+\theta}{\sigma}+1+\frac{\theta}{\sigma}\right] u_{o}^{\prime}\left(\omega^{o}+\left(1+\theta \sigma^{-1}\right) q_{*}(\sigma)\right) \pi_{\theta} \\
& =-\frac{1-\sigma}{\sigma} \sum_{\theta \in \Theta} u_{o}^{\prime}\left(\omega^{o}+\left(1+\theta \sigma^{-1}\right) q_{*}(\sigma)\right) \pi_{\theta}\left\{\begin{array}{c}
< \\
= \\
>
\end{array}\right\} 0 \text { if } \sigma\left\{\begin{array}{c}
< \\
= \\
>
\end{array}\right\} 1
\end{aligned}
$$

for each $\sigma \in\left[\underline{\theta}, r_{0}^{-1}[\right.$, where the second equality follows from Eq.(2). Here, we should note that, in the absence of nominal idiosyncratic risks (i.e.: $0 \in \Theta$ and $\pi_{0}=1$ ), which is the case excluded in the current setting, $V_{1}(\sigma, q)=0$ and $W^{\prime}(\sigma)=V_{2}\left(\sigma, q_{*}(\sigma)\right) q_{*}^{\prime}(\sigma)$. In that case, furthermore, the second-best money growth rate becomes $\sigma=1$, a stationary monetary equilibrium at which also becomes GRO and therefore is CPO. This may indicate that the term $V_{2}\left(\sigma, q_{*}(\sigma)\right) q_{*}^{\prime}(\sigma)$ is related to the intertemporal consumption smoothing and $\sigma \neq 1$ distorts the consumption smoothing between consumptions when young and old. On the other hand, in the presence of nominal idiosyncratic risks, we have shown that $V_{1}(\sigma, q)>0$, which is a factor increasing the equilibrium welfare. This is because an increase in $\sigma$ smooths contingent consumptions in the second-period (See also Eq.(1)). In other words, $V_{1}\left(\sigma, q_{*}(\sigma)\right)$ is related to the risk sharing in the second period and a low $\sigma$ distorts the risk sharing among contingent consumptions when old. Therefore, we can consider that $W^{\prime}(\sigma)=V_{1}\left(\sigma, q_{*}(\sigma)\right)+V_{2}\left(\sigma, q_{*}(\sigma)\right) q_{*}^{\prime}(\sigma)$ contains a trade-off between intertemporal consumption smoothing and better risk sharing and the central bank should choose a money growth rate to remedy such a trade-off. Then, the final proposition of this article provides a characterization of second-best monetary growth rates:

Proposition 5 A second-best money growth rate exists and is greater than one if and only if $r_{0}<1$. 
This proposition says that, in the presence of nominal idiosyncratic risks, a constant or reducing monetary policy is not optimal and there exists some expansionary monetary policy being optimal (more precisely, second best), provided that $r_{0}<1$, which means Pareto suboptimality of the initial endowment stream (See Proposition 1). The last condition makes money essential, i.e.: it ensures the existence of stationary monetary equilibrium even when there is no money growth, i.e.: $\sigma=1$.

To close this section, we presents an example illustrating some comparative statistics.

Example. Suppose that $\omega^{y}>0, \omega^{o}=0$, and $u_{y}(x)=u_{o}(x)=x^{1-\gamma} /(1-\gamma)$ for $\gamma>0$ where $u_{y}(x)=u_{o}(x)=\ln x$ if $\gamma=1$. First note that $\tau$ and $\rho$ are not independent of each other. To observe this fact, let $\left.\mu=\pi_{\rho} \in\right] 0,1[$. By the proviso that $-\tau(1-\mu)+\rho \mu=0$, we can obtain that $\tau(\rho):=\rho \mu /(1-\mu)=\tau \in] 0,1]$. Also note that it must hold that $\rho \leq(1-\mu) / \mu$ because $\tau(\rho) \leq 1$

In this economy, a monetary equilibrium given $\sigma$, denoted by $\left\{q_{t}(\sigma)\right\}_{t \geq 1}$, is characterized by

$$
(\forall t \geq 1) \quad q_{t+1}(\sigma)=\left[A(\sigma) \frac{\left(\omega^{y}-q_{t}(\sigma)\right)^{\gamma}}{q_{t}(\sigma)}\right]^{-\frac{1}{1-\gamma}} \quad \text { given } \quad q_{1}(\sigma)
$$

if $\gamma \neq 1$ and $q_{t}(\sigma)=A(\sigma) \omega^{y} /(1+A(\sigma))$ for all $t \geq 1$ if $\gamma=1$, where

$$
A(\sigma)=\frac{(1-\mu)(1-\tau(\rho))}{(\sigma-\tau(\rho))^{\gamma}}+\frac{\mu(1+\rho)}{(\sigma+\rho)^{\gamma}} .
$$

A unique stationary monetary equilibrium given $\sigma$, denoted by $q_{*}(\sigma)$, has then a closed form that

$$
q_{*}(\sigma)=\frac{[A(\sigma)]^{\frac{1}{\gamma}}}{1+[A(\sigma)]^{\frac{1}{\gamma}}} \omega^{y} .
$$

Here, we examine the effect of an increase in risk of nominal shocks on the welfare and the second-best money growth rate. Further specify the economy by $\omega^{y}=1$ (and $\omega^{o}=0$ ), $\gamma=0.5$, $\mu=0.5$. Because $\mu=0.5$, an increase in $\rho=\tau \in] 0,1]$ represents an increase in risk of nominal shocks in the sense of Rothschild and Stiglitz (1970). Figure 1 depicts the relation between money growth rate $\sigma$ and equilibrium welfare $W(\sigma)$ by the solid line when $\rho=0.45$, the dashed line when $\rho=0.65$, and the dot-dashed line when $\rho=0.85$. It can be observed that an increase in risk of nominal shocks worsens the welfare at a second-best money growth rate. In fact, we can calculate that the second-best money growth rate is about 1.25 if $\rho=0.45$, about 1.42 if $\rho=0.65$, and about 1.62 if $\rho=0.85$. The welfare at the second-best money growth rate is then 


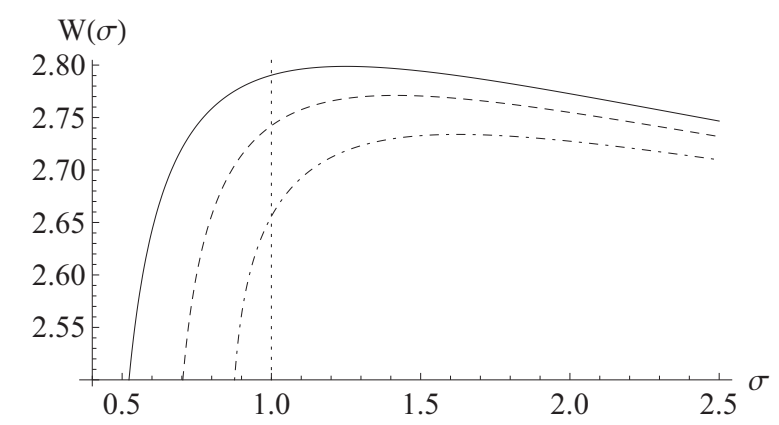

Figure 1: Money Growth Rate and Welfare
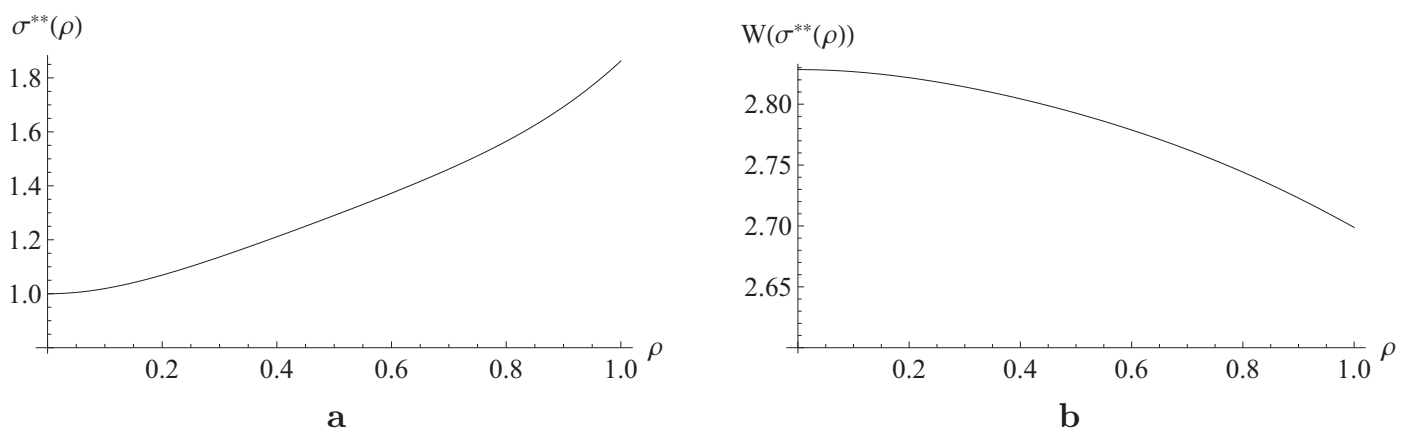

Figure 2: Comparative Statistics w.r.t. $\rho$

about 2.80 if $\rho=0.45$, about 2.77 if $\rho=0.65$, and about 2.73 if $\rho=0.85$. Figure 2.a depicts the relation between $\rho$ and the second-best money growth rate, denoted by $\sigma^{* *}(\rho)$ and Figure 2.b does between $\rho$ and the welfare at the second-best money growth rate. Therefore, an increase in risk of unexpected redistribution of money actually worsens the welfare.

\section{Proofs of Propositions}

This section provides proofs of propositions in the previous section.

Proof of Proposition 1. Let $c=\left(c^{y},\left(c^{\theta}\right)_{\theta \in \Theta},\left(c_{1}^{\theta}\right)_{\theta \in \Theta}\right)$ be an interior stationary feasible allocation. Let $\Theta(c):=\left\{\theta \in \Theta: c_{1}^{\theta}>0\right\}$. We first claim that $\Theta(c) \neq \emptyset$, i.e.: there exists some $\theta^{\prime} \in \Theta$ such that $c_{1}^{\theta^{\prime}}>0$. In fact, it follows from the fact that $c$ is interior that $0<\sum_{\theta \in \Theta} c^{\theta} \pi_{\theta}=$ $\sum_{\theta \in \Theta} c_{1}^{\theta} \pi_{\theta}$, which implies the existence of $\theta^{\prime} \in \Theta$ such that $c_{1}^{\theta^{\prime}}>0$.

We then characterize Pareto optimality. Note that $c$ is Pareto optimal if and only if there exist Pareto weights $\gamma \in \Re_{++}$and $\left(\gamma_{1}^{\theta}\right)_{\theta \in \Theta} \in \Re_{+}^{\Theta}$ such that $c$ maximizes $\gamma U\left(c^{y},\left(c^{\theta}\right)_{\theta \in \Theta}\right)+\sum_{\theta \in \Theta} \gamma_{1}^{\theta} c_{1}^{\theta} \pi_{\theta}$ subject to the resource constraints: $c^{y}+\sum_{\theta \in \Theta} c^{\theta} \pi_{\theta}=\omega^{y}+\omega^{o}$ and $c^{y}+\sum_{\theta \in \Theta} c_{1}^{\theta} \pi_{\theta}=\omega^{y}+\omega^{o}$. Therefore, Pareto optimality of $c$ is completely characterized by the existence of Pareto weights 
$\gamma \in \Re_{++}$and $\left(\gamma_{1}^{\theta}\right)_{\theta \in \Theta} \in \Re_{+}^{\Theta}$ and Lagrange multipliers $\lambda \geq 0$ and $\lambda_{1} \geq 0$ such that

$$
\gamma u_{y}^{\prime}\left(c^{y}\right)=\lambda+\lambda_{1}
$$

$$
(\forall \theta \in \Theta) \quad \gamma u_{o}^{\prime}\left(c^{\theta}\right)=\lambda, \quad \text { and }
$$

$$
(\forall \theta \in \Theta) \quad \gamma_{1}^{\theta} \leq \lambda_{1} \quad \text { with equality if } \quad c_{1}^{\theta}>0 .
$$

Note that $\lambda_{1}=\gamma_{1}^{\theta^{\prime}}$ for each $\theta^{\prime} \in \Theta(c)$ because $c_{1}^{\theta^{\prime}}>0$.

Suppose that there exists $\gamma \in \Re_{++},\left(\gamma_{1}^{\theta}\right)_{\theta \in \Theta} \in \Re_{+}^{\Theta}, \lambda \geq 0$, and $\lambda_{1} \geq 0$ satisfying Eqs.(3)-(5). By Eq.(4), there exists some $c^{o}>0$ such that $c^{o}=c^{\theta}$ for each $\theta \in \Theta$. By Eqs.(3) and (4), it follows that

$$
1=\frac{u_{o}^{\prime}\left(c^{o}\right)}{u_{y}^{\prime}\left(c^{y}\right)}+\frac{\lambda_{1}}{\gamma u_{y}^{\prime}\left(c^{y}\right)} \geq \frac{u_{o}^{\prime}\left(c^{o}\right)}{u_{y}^{\prime}\left(c^{y}\right)}
$$

because $\lambda_{1} \geq 0$.

On the other hand, suppose the existence of $c^{o}>0$ such that $c^{o}=c^{\theta}$ for each $\theta \in \Theta$ and $u_{o}^{\prime}\left(c^{o}\right) / u_{y}^{\prime}\left(c^{y}\right) \leq 1$. Let $\gamma=1, \lambda=u_{o}^{\prime}\left(c^{o}\right), \lambda_{1}=\gamma_{1}^{\theta^{\prime}}=u_{y}^{\prime}\left(c^{y}\right)-u_{o}^{\prime}\left(c^{o}\right) \geq 0$ for each $\theta^{\prime} \in \Theta(c)$ and $\gamma_{\theta^{\prime \prime}}^{1}=0$ for each $\theta^{\prime \prime} \in \Theta \backslash \Theta(c)$. It is immediate to verify that $\gamma \in \Re_{++},\left(\gamma_{1}^{\theta}\right)_{\theta \in \Theta} \in \Re_{+}^{\Theta}$, $\lambda \geq 0$, and $\lambda_{1} \geq 0$ satisfies Eqs.(3)-(5). This completes the proof of characterization of Pareto optimality.

Finally, we characterize golden rule optimality. Note that $c$ is golden rule optimal if and only if it maximizes $U\left(c^{y},\left(c^{\theta}\right)_{\theta \in \Theta}\right)$ subject to the resource constraints: $c^{y}+\sum_{\theta \in \Theta} c^{\theta} \pi_{\theta}=\omega^{y}+\omega^{o}$ and $c^{y}+\sum_{\theta \in \Theta} c_{1}^{\theta} \pi_{\theta}=\omega^{y}+\omega^{o}$. Then, the solution of the previous optimization problem is completely characterized by, in addition to the stationary feasibility of $c, u_{y}^{\prime}\left(c^{y}\right)=u_{o}^{\prime}\left(c^{\theta}\right)$ for each $\theta \in \Theta$. Because of strict concavity of $u_{o}$, the last condition is equivalent to the existence of some $c^{o}>0$ such that $c^{o}=c^{\theta}$ for each $\theta \in \Theta$ and $u_{o}^{\prime}\left(c^{o}\right) / u_{y}^{\prime}\left(c^{y}\right)=1$. This completes the proof of Proposition 1.

Q.E.D.

Proof of Proposition 2. Combining the first-order conditions of the agents' optimization problems and the money market clearing condition, we can obtain Eq.(1).

Q.E.D.

Proof of Proposition 3. Let $\sigma \geq \tau$. Because $\underline{\theta}<0, \sigma>0$. Note that $1+\theta \sigma^{-1} \geq 0$ for each $\theta \in \Theta$. In fact, for each $\theta<0$, we obtain that $1+\theta \sigma^{-1} \geq 1+\underline{\theta} \sigma^{-1} \geq 0$. On the other hand, for each $\theta \geq 0,1+\theta \sigma^{-1} \geq 1 \geq 0$. 
Define the function $f:] 0, \omega^{y}[\rightarrow \Re$ by

$$
(\forall q \in] 0, \omega^{y}[) \quad f(q):=\frac{u_{y}^{\prime}\left(\omega^{y}-q\right)}{\sum_{\theta \in \Theta}(1+\theta) u_{o}^{\prime}\left(\omega^{o}+\left(1+\theta \sigma^{-1}\right) q\right) \pi_{\theta}} .
$$

Note that $f$ is continuous. Furthermore, $f$ is strictly monotone increasing because it follows that, for each $q \in] 0, \omega^{y}[$,

$$
f^{\prime}(q)=\frac{-u_{y}^{\prime \prime}\left(c^{y}\right) \sum_{\theta \in \Theta}(1+\theta) u_{o}^{\prime}\left(c^{\theta}\right) \pi_{\theta}-u_{y}^{\prime}\left(c^{y}\right) \sum_{\theta \in \Theta}(1+\theta)\left(1+\theta \sigma^{-1}\right) u_{o}^{\prime \prime}\left(c^{\theta}\right) \pi_{\theta}}{\left(\sum_{\theta \in \Theta}(1+\theta) u_{o}^{\prime}\left(c^{\theta}\right) \pi_{\theta}\right)^{2}}
$$

and $1+\theta \sigma^{-1} \geq 0$, where $c^{y}=\omega^{y}-q$ and $c^{\theta}=\omega^{o}+\left(1+\theta \sigma^{-1}\right) q$ for each $\theta \in \Theta$.

First suppose the existence of stationary monetary equilibrium, $q \in] 0, \omega^{y}[$. By strict monotonicity of $f$, we can obtain that $1 / \sigma=f(q)>\lim _{x \downarrow 0} f(x)=u_{y}^{\prime}\left(\omega^{y}\right) / u_{o}^{\prime}\left(\omega^{o}\right)$.

Suppose now that $u_{y}^{\prime}\left(\omega^{y}\right) / u_{o}^{\prime}\left(\omega^{o}\right)<1 / \sigma$. Because $\lim _{q \downarrow 0} f(q)<1 / \sigma$ and $\lim _{q \uparrow \omega^{y}} f(q)=\infty>$ $1 / \sigma$, it follows from the intermediate value theorem that there exists some $q \in\left(0, \omega^{y}\right)$ such that $f(q)=1 / \sigma$. This $q$ is stationary monetary equilibrium.

By strict monotonicity of $f$, we can conclude such a $q$ is unique. This completes the proof of Proposition 3.

Q.E.D.

Proof of Proposition 4. Let $\sigma \in\left[\tau, r_{0}^{-1}\right.$ [. Then, the consumption stream at the equilibrium, denoted by $\left(c^{y},\left(c^{\theta}\right)_{\theta \in \Theta}\right)$, is given by $c^{y}=\omega^{y}-q_{*}(\sigma)$ and $c^{\theta}=\omega^{o}+\left(1+\theta \sigma^{-1}\right) q_{*}(\sigma)$ for each $\theta \in \Theta$. By the proviso on the probability space, $(\Theta, \pi)$, there exists at least one $\hat{\theta}>0$ in $\Theta$. Obviously, $c^{\underline{\theta}} \neq c^{\hat{\theta}}$, which does not satisfy the characterization of Pareto optimality provided by Proposition 1 . Therefore, the stationary monetary equilibrium given $\sigma$ cannot achieve a Pareto optimal allocation.

Q.E.D.

Proof of Proposition 5. Suppose the existence of a second-best money growth rate $\sigma^{*}$, which exceeds unity. Then, it follows from its definition that $1<\sigma^{*}<r_{0}^{-1}$, which implies that $r_{0}<1 / \sigma^{*}<1$. This completes the only if part of the proposition.

Suppose now that $r_{0}<1$ or equivalently $1<r_{0}^{-1}$. Recall that $q_{*}^{\prime}(\sigma)<0, V_{1}(\sigma, q)>0$, and

$$
V_{2}\left(\sigma, q_{*}(\sigma)\right)\left\{\begin{array}{c}
< \\
= \\
>
\end{array}\right\} \quad 0 \text { if } \sigma\left\{\begin{array}{c}
< \\
= \\
>
\end{array}\right\} 1
$$

for each $q>0$ and each $\sigma \in\left[\tau, r_{0}^{-1}[\right.$. Thus, we can obtain that

$$
W^{\prime}(\sigma)=V_{1}\left(\sigma, q_{*}(\sigma)\right)+V_{2}\left(\sigma, q_{*}(\sigma)\right) q_{*}^{\prime}(\sigma)>0
$$


for each $\sigma \in[\tau, 1]$, which implies that a second-best money growth rate, if any, is greater than one. Furthermore, it follows from the fact that $\lim _{\sigma \uparrow r_{0}^{-1}} q_{*}(\sigma)=0$ and $r_{0}<1$ that

$$
\lim _{\sigma \uparrow r_{0}^{-1}} W^{\prime}(\sigma)=\lim _{\sigma \uparrow r_{0}^{-1}} V_{2}\left(\sigma, q_{*}(\sigma)\right) q_{*}^{\prime}(\sigma)<0,
$$

which implies that there exists some $\delta \in] 0, r_{0}^{-1}-1\left[\right.$ such that $W^{\prime}\left(r_{o}^{-1}-\varepsilon\right)<0$ for each $\left.\left.\varepsilon \in\right] 0, \delta\right]$. Therefore, every $\sigma \in\left[r_{0}^{-1}-\delta, r_{0}^{-1}[\right.$ is not second-best and a second-best money growth rate, if any, is less than $\bar{\sigma}:=r_{0}^{-1}-\delta>1$.

As a summary, a second-best money growth rate, if any, lies on $[1, \bar{\sigma}]$. Because $W$ is continuous on $[1, \bar{\sigma}]$, which is a compact set, we can conclude that there exists a second-best money growth rate $\sigma^{* *}$ in $[1, \bar{\sigma}]$. Finally, it can be shown by applying the intermediate value theorem that $\left.\sigma^{* *} \in\right] 1, \bar{\sigma}\left[\right.$ because $W^{\prime}(1)>0$ and $W^{\prime}(\sigma)<0$.

Q.E.D.

\section{Acknowledgements}

This article was financially supported by a grant-in-aid from Keizai Boueki Kenkyujo. The author thanks to Professor Yasuo Maeda for his supervision and seminal participants of 2013 Japan Society of Monetary Economics Autumn Meeting at Nagoya University for their helpful comments.

\section{References}

Akyol, A. (2004) "Optimal monetary policy in an economy with incomplete markets and idiosyncratic risk," Journal of Monetary Economics 51, 1245-1269.

Bhattacharya, J., J. Haslag, and A. Martin (2005) "Heterogeneity, redistribution, and the Friedman rule," International Economic Review 46, 437-454.

Bhattacharya, J., J. Haslag, and A. Martin (2009) "Optimal monetary policy and economic growth," European Economic Review 53, 210-221.

Friedman, M. (1969) "The optimum quantity of money," in The Optimum Quantity of Money and Other Essays, Aldine: Chicago.

Haslag, J.H. and A. Martin (2007) "Optimality of the Friedman rule in an overlapping generations model with spatial separation," Journal of Money, Credit and Banking 39, 1741-1758. 
Kryvtsov, O., M. Shukayev, and A. Ueberfeld (2011) "Optimal monetary policy under incomplete markets and aggregate uncertainty: A long-run perspective," Journal of Economic Dynamics and Control 35, 1045-1060.

Ohtaki, E. (2013) "Golden rule optimality in stochastic OLG economies," Mathematical Social Sciences 65, 60-66.

Gomis-Porquears, P. and B.D. Smith (2003) "Seasonality and monetary policy," Macroeconomic Dynamics 7, 477-502.

Rothschild, M., Stiglitz, J.E. (1970) "Increasing risk: I. A definition," Journal of Economic Theory 2, 225-243.

Smith, B.D. (2002) "Monetary policy, banking crises, and the Friedman rule," American Economic Review Papers and Proceedings 92, 128-134.

Zhu, Tao (2008) "An overlapping generations model with search," Journal of Economic Theory 142, 318-331. 